\title{
12. The effectiveness of Bilateral Erector Spinae Plane Block in reducing perioperative opioid administration in patients undergoing Laparoscopic Cholecystectomy
}

Freideriki Sifaki ${ }^{1,2, *}$, Ioannis Mantzoros ${ }^{3}$, Eleni Koraki², Stella Bagntasarian², Panagiotis Christidis ${ }^{3}$, Vaia Tsapara ${ }^{2}$, Kassiani Theodoraki ${ }^{1}$

${ }^{1} 1^{\text {st }}$ University Department of Anesthesiology, “Aretaieio Hospital”, National and Kapodistrian University of Athens, Athens, Greece; ${ }^{2}$ Anesthesiology Clinic of General Hospital of Thessaloniki "Georgios Papanikolaou", Thessaloniki, Greece; ${ }^{3} 4^{\text {th }}$ University Department of Surgery, General Hospital of Thessaloniki "Georgios Papanikolaou", Aristotle University of Thessaloniki, Thessaloniki, Greece.*frida1sif1@gmail.com

Introduction: Laparoscopic cholecystectomy (LC), is one of the most common surgeries performed in general surgery. Most of the times, LC is accompanied by moderate to severe postoperative pain. Erector Spinae Plane Block (ESPB) is an innovative trunk block which has been used as a method of postoperative analgesia in various clinical procedures. In this study we evaluated its effectiveness as a method of perioperative analgesia, seeking to investigate whether it is effective in reducing perioperative opioid administration in patients undergoing LC.

Methods: This is a double-blind, randomized, controlled, prospective study. 60 patients were randomized into Group C (ESPB with N/S 0.9\%), Group D (ESPB with ropivacaine 0.375\%, dexmedetomidine $1 \gamma / \mathrm{kg}$ ) and Group R (ESPB with ropivacaine $0.375 \%$ ). ESPB was performed bilaterally before induction of general anesthesia, with ultrasound guidance. Statistical analysis included ANOVA, two-way ANOVA for repeated measures, Kruskal-Wallis and Spearman tests.

Results: All patients remained hemodynamically stable during their hospitalization, without major complications. Statistical significance was found to exist regarding total perioperative remifentanil consumption between all three Groups. Median morphine consumption, NRS pain scores and mobilization time of the patients was found to be significantly less in patients of Group D compared with patients of Group C and in patients of Group R compared with patients of Group C. However, there was no statistically important difference between Groups D and R. Satisfaction scores were found to be statistically higher in patients of Group D when compared with patients of Group C.

Conclusion: ESPB performance with administration of ropivacaine or a combination of ropivacaine and dexmedetomidine in patients undergoing LC, is an innovative, safe and simple method which contributes to the amelioration of the quality of perioperative analgesia, avoiding the complications arising from opioid administration and thus, achieving multimodal analgesia. 